\title{
Design and Simulation of Indoor Tour Guide Robot Based on ROS
}

\author{
Yuheng Zhang ${ }^{1,2}$, Yizhun Peng ${ }^{12^{*}}$, Lianchen zhao ${ }^{1,2}$ \\ ${ }^{1}$ College of Electronic Information and Automation, \\ Tianjin University of Science and Technology, China \\ ${ }^{2}$ Advanced Structural Integrity International Joint Research Centre, \\ Tianjin University of Science and Technology, China

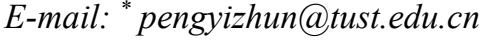 \\ www.tust.edu.cn
}

\begin{abstract}
Aiming at the problems of the small number of lecturers in museums and other venues and the large demand for audience consultation, a ros-based indoor guide robot was designed. The robot consists of a mechanical system, a motion control system, and a sensor system. In order to improve the efficiency of development and debugging, and reduce the cost of experiments, it is necessary to test related algorithms in a virtual simulation environment before the robot enters the actual working state. Experiments have proved that the robot can autonomously guide guests to the destination and explain according to a preset path; the robot has multiple sensors to sense obstacles, and can autonomously avoid obstacles during the explanation and continue to move; the robot can accurately and efficiently recognize faces and provide accurate services Fast.
\end{abstract}

Keywords: Indoor tour guide robot; Autonomously guide; Voice interaction; Face recognition

\section{Introduction}

With the rapid development of intelligent technology, the quality of people's lives continues to improve. Since the existing "guide" intelligent robots can only realize the function of navigating the road when in use, the function is relatively single, and the existing "guide" intelligent robots cannot guide tourists and cannot achieve the effect of reminding. ${ }^{1}$ Aiming at the problems of museums and other venues such as the lack of interpreters and the large demand for audience consultation, a ros-based indoor tour guide robot was designed and simulated in gazebo software. The robot mainly realizes the following 3 functions:

(i) Welcome explanation: The robot can guide the guests to the destination and give an explanation according to a preset path. After the explanation, the robot will automatically return to the original starting position;

(ii) Audio guide: The robot can interact with people by voice. During the robot's explanation process, the audience can interact with the robot at any time if they have questions or problems.

(iii) Face recognition: The robot takes pictures of the user who is guided for the first time to collect public information, continuously enriching the public's personal portraits, establishing more complete personal information materials, and providing accurate and fast services.

\section{The Composition of the Robot}

The robot consists of four layers. The first layer is the chassis movement layer, including two motors, two driving wheels, one universal wheel, and three infrared sensors; the second layer is a lidar layer with a lidar; the third layer It is the driving layer, including the lower computer, battery, three ultrasonic sensors, a smoke sensor, and a temperature and humidity sensor; the fourth layer is the decision-making computing layer, including the upper computer, monitor, camera, microphone and speaker. The schematic diagram of the robot is shown in Figure 1.The upper computer is an industrial computer with high anti-

(C) The 2021 International Conference on Artificial Life and Robotics (ICAROB2021), January 21 to 24, 2021 
magnetic, dust-proof and impact-proof capabilities. ${ }^{2}$ There is a special power supply in the chassis, and the power supply has strong anti-interference ability. The processor is an inter 17 processor with four usb3.0 interfaces And four usb2.0 interfaces, two network ports. The lower computer controller mainly realizes the bottom-level motor drive, power management and acquisition of various sensors such as ultrasonic and infrared, and at the same time feeds back the collected data to the upper computer in time. The lower computer controller uses STMicroelectronics' STM32F103ZET6 chip as the main control, the chip flash has $512 \mathrm{~K}$, the SRAM is $64 \mathrm{~KB}$, and the pins are $144 .^{3}$ The chip runs the FreeRTOS system. The infrared sensor can realize the function of assisting obstacle avoidance, preventing the robot from falling when it reaches the road pit. ${ }^{4}$ Lidar is used to realize the range finding scan and generate the plane point cloud map information of the space. These cloud map information can be used in practical applications such as map surveying, robot positioning and navigation, and object/environment modeling. The block diagram of the robot system is shown in Figure 2.

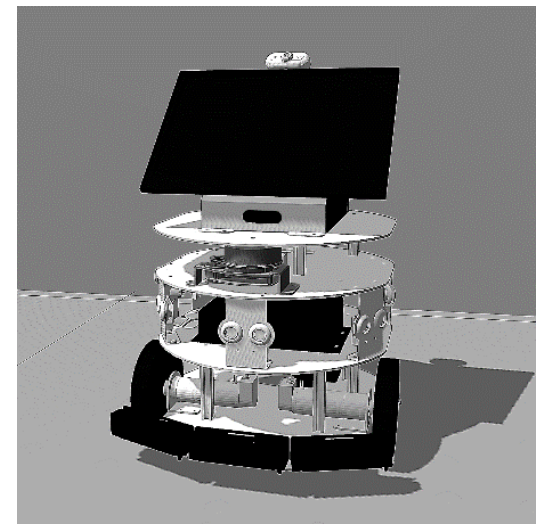

Fig.1. The schematic diagram of the robot

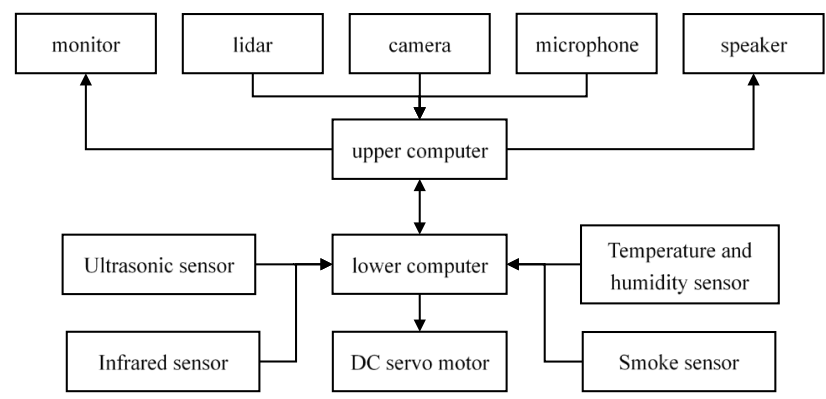

Fig.2. The block diagram of the robot system

\section{Robot Simulation Test}

\subsection{Construction of simulation environment}

The simulation environment of the robot is built on the gazebo simulation platform, and the walls are drawn by the building editor tool provided by gazebo. We built the indoor scene of a simulated exhibition hall, including venues in 16 Chinese cities such as Beijing and Tianjin. Each venue is 12 meters long and 4 meters wide. The robot model is built and exported by solidworks software. The initial position of the robot is in the center of the hall of the exhibition hall. The simulation environment of the robot is shown in Figure 3.

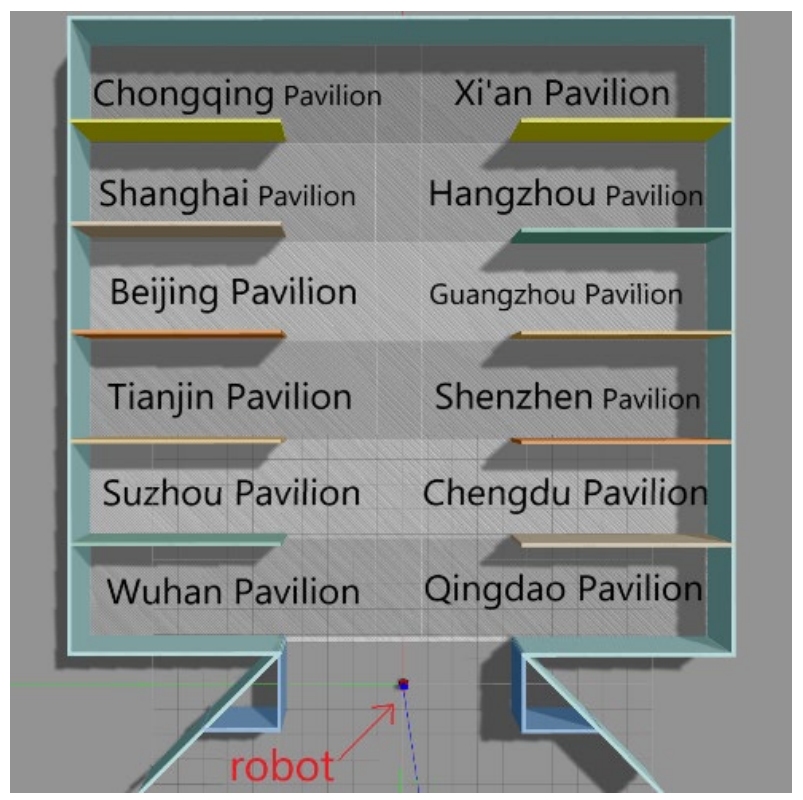

Fig.3. Robot simulation environment

\subsection{Mapping and navigation}

Only after the establishment of the map is completed and the map is obtained, the robot can be controlled to navigate. SLAM is simultaneous localization and mapping, which can be described as: the robot starts to move from an unknown position in an unknown environment. ${ }^{5}$ During the movement, it locates itself according to the position estimation and the map, and at the same time builds an incremental map to realize the robot's autonomous positioning and navigation. ${ }^{6}$ This design uses the most commonly used and mature gmapping algorithm in SLAM, gmapping integrates the Rao-Blackwellized particle filter

(C) The 2021 International Conference on Artificial Life and Robotics (ICAROB2021), January 21 to 24, 2021 
algorithm, the following is the overall framework of gmapping and navigation.

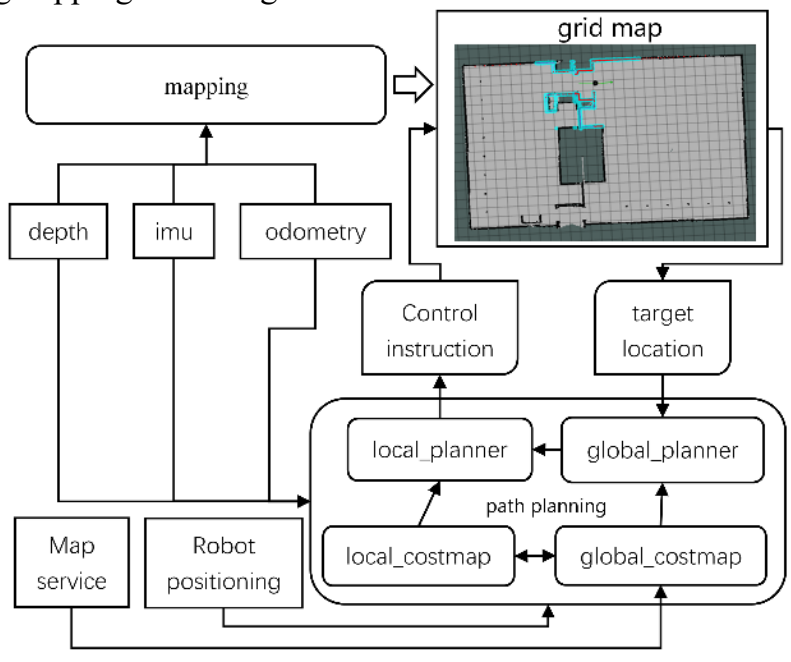

Fig.4. The overall framework of gmapping and navigation

The gmapping function package subscribes to the robot's depth information, IMU information and odometry information, and at the same time completes the configuration of some necessary parameters, you can create and output a probability-based two-dimensional grid map. The key to navigation is robot positioning and path planning. The navigation function package needs to collect the sensor information of the robot to achieve the effect of real-time obstacle avoidance. It also needs the odometry information of the robot and the corresponding $\mathrm{TF}$ transformation. Finally, the navigation function package outputs instructions that can control the movement of the robot.

Use the keyboard node to control the robot to move in the Gazebo environment. After the map is created, save it.This map will be called during the navigation process.

Use the move base function package for global path planning and local real-time planning. According to the given target location and the constructed global map, the $\mathrm{A}^{*}$ algorithm is used to plan the global path, and the optimal route from the robot to the target location is calculated as the global route. In practice, obstacles may appear at any time, so local real-time planning must be carried out to make it conform to the global optimal path as much as possible. Use the Dynamic Window Approaches algorithm to search for multiple paths to avoid and travel, and select the optimal path according to the evaluation index. Use the amcl function package to complete the autonomous positioning function, that is, the robot can calculate its position on the map in any state. ${ }^{7}$ Perform navigation simulation in Rviz, as shown in the figure below, where the red arrow is the specified target position, and the green line between the red arrow and the robot is the global path planned by the navigation function package.

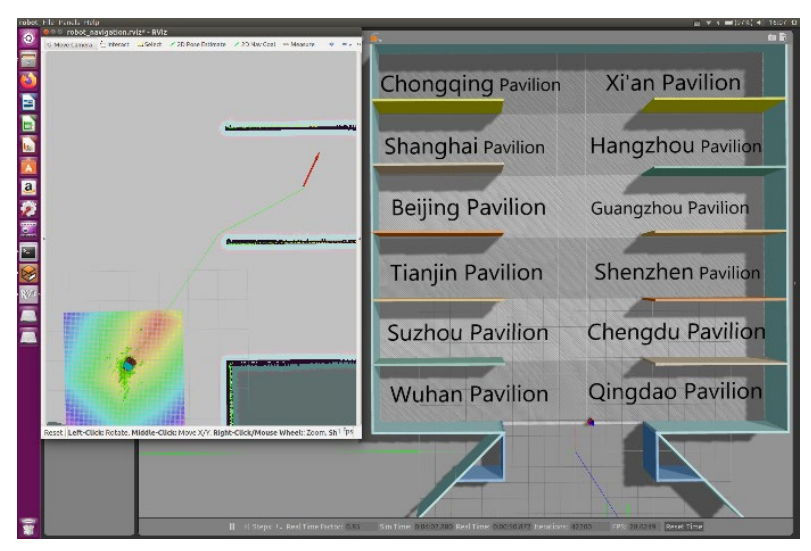

Fig.5. Robot navigation simulation

\subsection{Welcome explanation}

The robot can guide the guests to the destination and give an explanation according to a preset path. After the explanation, the robot will automatically return to the original starting position. When building a map, record the location of each venue as a navigation target point. Move_base implements path planning through action, and action uses a client/server architecture. Use the send goal() interface to send to the server of move base. After receiving the target task, the server will realize the path planning function according to the current pose of the robot, and output the speed command to control the movement of the robot. Go to the target location and return the result to the client. If the result is successful, it will send the introduction of the corresponding venue to the speech synthesis module, and let the robot speak the introduction. This function module is based on the tts_sample program in the iFLYTEK SDK, modify the main code file, add the ros interface, subscribe to the introduction topic, receive the input voice string, and use the SDK interface in the callback function to convert the string into Voice, after the voice broadcast is completed, the robot will navigate to the next target point and continue to broadcast until the last target point is finished. 


\subsection{Audio guide}

This function is based on the speech recognition SDK provided by IFLYTEK and is constructed using the ROS system. The iat_record_sample program in IFLYTEK SDK is modified and the required ROS interface is added. This design adds a publisher, a subscriber and a move_base client. The Subscriber is used to receive the voice wake-up signal. After receiving the wake-up signal, the wakeupFlag variable is set, and then the voice dictation function of the SDK is called in the main loop to recognize Human voice information, set resultFlag variable after successful recognition. Then use dataString.find() to determine the meaning of Chinese voice input, and publish the corresponding target point pose through the send_goal() interface. After receiving the target task, the server of move base will realize global and local Path planning function according to the current pose of the robot, output speed command to control the robot to move to the target position, and return the result to the client. If the result is successful, it will send the introduction of the corresponding venue to the speech synthesis module, and let the robot speak the introduction.

\subsection{Target face recognition}

The specific process of face detection is shown in Figure 6. First, load the face detection classifier that comes with opencv. In this article, the classifier based on haar feature values is used. In this article, we mainly use the contour of the face and human eye recognition to realize face detection. The next step is to preprocess the image to be recognized, to grayscale the image, obtain its histogram, and then perform equalization. This step is mainly to make the feature value of the face in the image better extracted come out. Calling the detectMultiscal function to recognize the picture is mainly because the input picture is of different sizes, so it needs to be detected in multiple dimensions. ${ }^{8}$ By calling the rectangle function, draw the rectangular frame of the face profile recognized by the program. Finally, the drawn rectangular frame of the face is cut out in the original image.

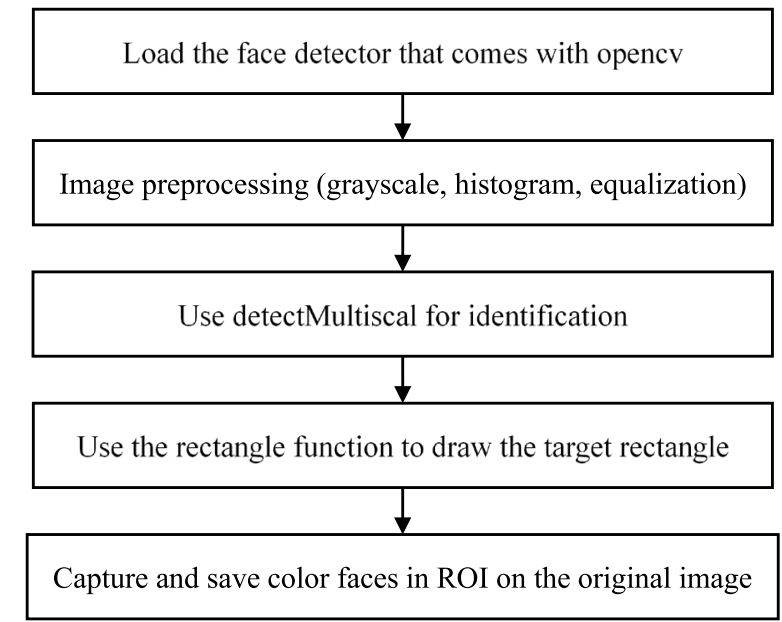

Fig.6. Face detection flowchart

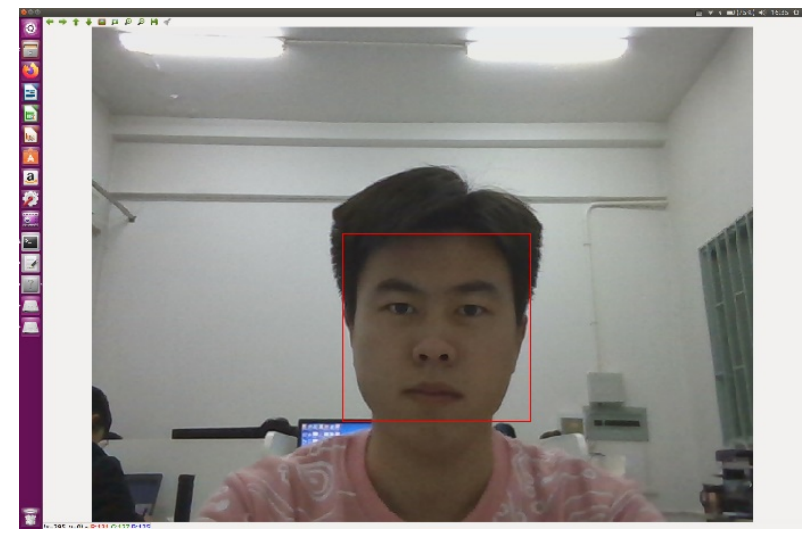

Fig.7. Face detection

To let the program know who to recognize, it is necessary to train the face model. Firstly, the target image is preprocessed, and the sample is normalized, mainly for the same size of the image, and then grayscale. Next, label the sample, this is to tell the training model which number belongs to whose face. Then start training, mainly using three methods, namely EigenFaceRecognizer, FisherFaceRecognizer and LBPHFaceRecognizer. The EigenFaceRecognizer method is also face recognition based on PCA transformation. The principle of PCA transformation is a way to reduce the dimensionality of an image, because in face recognition, the image is generally processed as a vector, but at the same time, the dimensionality of the vector is too large, and the huge dimensionality is quite difficult for subsequent image calculations. Therefore, it is necessary to reduce the image dimension without losing important information as much 
as possible. The FisherFaceRecognizer method is a face recognition based on Fisher transform. ${ }^{9}$ LBPHFaceRecognizer is a face recognition method based on local binary patterns. After training, save the training model locally, and then you can start face recognition.

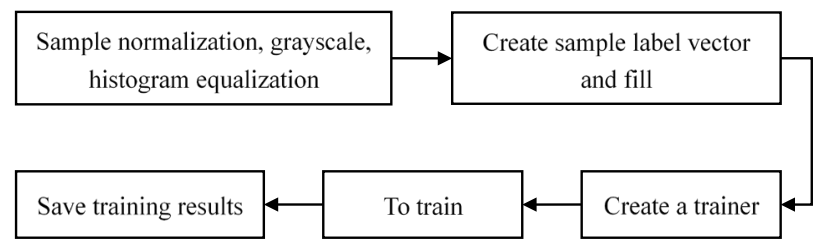

Fig.8. Face training flowchart

After having the above foundation, the final face recognition can be performed. First, image preprocessing is performed, and then the trained classifier is loaded to obtain the label value on the input image, and then the character name is obtained through the label value.

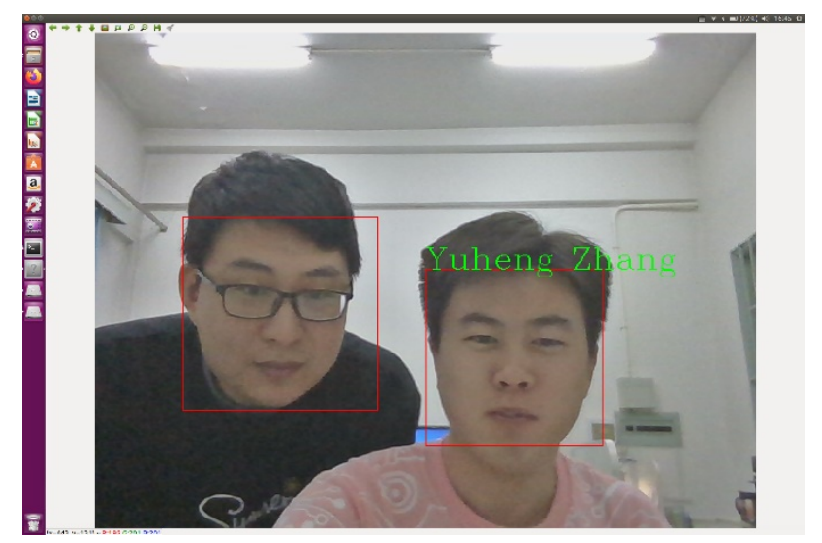

Fig.9. Target face recognition

\section{Conclusion}

In view of the small number of lecturers in museums and other venues and the large demand for audience consultation, the overall robot design was completed, and experiments were carried out on the gazebo simulation platform. The test proved that the robot can complete the functions of welcome explanation, voice guide, and face recognition. By interacting with robots, visitors can understand the situation of each exhibition area. The robot's way-guide function can also provide more convenience for visitors. This solution can improve the construction effectiveness and quality of museums and other venues, as well as a smart and personalized service system.

(C) The 2021 International Conference on Artificial Life and Robotics (ICAROB2021), January 21 to 24, 2021

\section{References}

1. Jianfa Dai, Jingrong Ying, Hongtao Liu. A "Guide" Intelligent Robot Design, Science Technology and Innovation, 2020(11): 142-143.

2. Wenbin Ni, Guanghua Lu, Yi Zhang. A guide service robot design , Machinery Manufacturing and Automation, 2016, 45(06): 176-179.

3. Jing He. Design and production of campus tour guide robot, Electronic Testing, 2018(11): 76-77.

4. Chenxi Guan. Design and research of indoor inspection robot system based on ROS, Electromechanical Information, 2020(30): 128-129.

5. Cadena C.,Carlone L.,Carrillo H.,et al.Past,Present,and Future of Simultaneous Localization and Mapping:Toward the Robust-Perception Age, IEEE Transactions on Robotics,2016,32(6):1309-1332.

6. Rowekamper J.,Sprunk C., Tipaldi G D.,et al.On the position accuracy of mobile robot localization based on particle filters combined with scan matching, Intelligent robots and systems, 2012:3158-3164.

7. Yanbin G.,Shifei L.,Mohamed A.,et al.INS/GPS/LiDAR Integrated Navigation System for Urban and Indoor Environments Using Hybrid Scan Matching Algorithm, Sensors, 2015,15(9):23286-23302.

8. Honglong Chen, Dongyong Liu, Zhichen Ni,et al.The face recognition experiment platform of quadrotor drone based on YOLO, Experimental Technology and Management, 2020,37(10):107-111.

9. REDMON J, DIVVALA S, GIRSHICK R, et al. You only look once:Unified, real-time object detection, in IEEE Conference on Computer Vision and Pattern Recognition(CVPR), Las Vegas, Nevada, USA, 2016:779788 . 